\title{
On the genera of polyhedral embeddings of cubic graphs.
}

\author{
Gunnar Brinkmann ${ }^{1} \quad$ Thomas Tucker $^{2} \quad$ Nico Van Cleemput ${ }^{1}$ \\ 1 Ghent University, Belgium \\ 2 Colgate University, USA
}

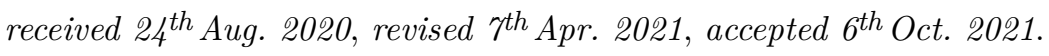

In this article we present theoretical and computational results on the existence of polyhedral embeddings of graphs. The emphasis is on cubic graphs. We also describe an efficient algorithm to compute all polyhedral embeddings of a given cubic graph and constructions for cubic graphs with some special properties of their polyhedral embeddings. Some key results are that even cubic graphs with a polyhedral embedding on the torus can also have polyhedral embeddings in arbitrarily high genus, in fact in a genus close to the theoretical maximum for that number of vertices, and that there is no bound on the number of genera in which a cubic graph can have a polyhedral embedding. While these results suggest a large variety of polyhedral embeddings, computations for up to 28 vertices suggest that by far most of the cubic graphs do not have a polyhedral embedding in any genus and that the ratio of these graphs is increasing with the number of vertices.

Keywords: polyhedron, graph, map, surface

\section{Introduction}

Three-dimensional convex polyhedra, or short polyhedra, as geometric objects have already been a subject of mathematical research in ancient Greece and played an important role ever since. Since Steinitz's theorem characterizing the graph formed by the edges of polyhedra as 3-connected plane graphs, these graphs - also referred to as polyhedra - are also studied as combinatorial objects. While the geometric characterization cannot directly be generalized to graphs on surfaces of higher genus, some key properties of polyhedra can be used to generalize the concept to polyhedral embeddings of higher genus:

Definition $1 A$ polyhedral embedding of a graph $G=(V, E)$ in an orientable surface is an embedding so that each facial walk is a simple cycle and an intersection of two faces is either empty, a single vertex, or a single edge.

An equivalent definition Mohar and Thomassen (2001) is that $G$ is 3 -connected and that the embedding has facewidth at least 3 (with the facewidth of a plane graph defined as infinite). For cubic embedded graphs, this is equivalent to the dual being simple - that is without multiedges

ISSN 1365-8050॰ 2021 by the author(s) Distributed under a Creative Commons Attribution 4.0 International License 
or loops. Like in most articles, in this article the word graph refers to simple graphs, but in some cases, like e.g. when talking about the dual, where the result of the operation might be a multigraph, we emphasize the requirement of being simple.

Polyhedral embeddings share many properties of polyhedra. For example, the dual of a polyhedral embedding is a polyhedral embedding Mohar (1997). Furthermore, local modifications of an embedding preserving symmetry, such as next to dual also e.g. truncation, ambo, and many others preserve polyhedrality of an embedding Van den Camp (2020). Finally, a generalization of Whitney's theorem that polyhedra have a unique embedding in the plane, shows that for each genus $g$, there is a number $\zeta(2 g)$ such that no graph can have more than $\zeta(2 g)$ polyhedral embeddings in the orientable surface of genus $g$ Mohar and Robertson (2001).

Cubic graphs are an especially interesting class of graphs, as for many more general conjectures it has been shown that it is sufficient to prove them for cubic graphs. Furthermore the dual of a cubic polyhedrally embedded graph is a simple triangulation. Triangulations of surfaces have a long history in topology and combinatorics: the Hauptvermutung of the 1920s, the Ringel-Youngs Theorem of the 1960s, or e.g Malnič and Mohar (1992); Negami (1999). On the other hand, most of that work does not analyze the structure of the cubic dual embedding.

We deal with combinatorial embeddings in closed oriented surfaces. We will first describe the basic concepts and notations. For further results and definitions, we refer the reader to Gross and Tucker (1987). For vertices $x, y$ of an embedded graph $G$, an edge $\{x, y\}$ is interpreted as two oppositely directed edges - the edge $(x, y)$ directed from $x$ to $y$ and the edge $(y, x)$ directed from $y$ to $x$. We refer to $(y, x)$ as the inverse edge of $(x, y)$ and write $e^{-1}$ for the inverse of the directed edge $e$. At each vertex we have a rotational order of the directed edges starting at that vertex, that we interpret in figures as clockwise. Such an assignment of cyclic orders is often called a rotation system. The mirror image of a combinatorial embedding assigns the reverse cyclic order for each vertex and represents the same topological embedding in the oppositely oriented surface. An isomorphism between two embedded graphs is a graph isomorphism that also respects the rotational order at each vertex or reverses the rotational order at each vertex, in which case we call the isomorphism orientation reversing. When we talk about the next (directed) edge of a directed edge $e$ (notation: $\mathrm{nx}(e)$ ), this refers to the next edge in rotational order around the start vertex of $e$. A face is a cyclic sequence $e_{0}, \ldots, e_{k-1}$ of directed edges, so that for $0 \leq i<k$ we have $\mathrm{nx}\left(e_{i}^{-1}\right)=e_{(i+1)(\bmod k)}$. In figures this corresponds to a counterclockwise traversal of the edges. We say that the set $\{e, \operatorname{nx}(e)\}$ of edges $e, \operatorname{nx}(e)$ forms an angle. In this case $e^{-1}, \operatorname{nx}(e)$ follow each other in a face of $G$ and $\operatorname{nx}(e)^{-1}, e$ follow each other in a face of the mirror image.

If we have a directed simple cycle $C=\left(e_{0}, \ldots, e_{k-1}\right)$ with $e_{i}=\left(v_{i}, v_{(i+1)}(\bmod k)\right)$ and a directed edge $e=\left(v_{i+1}, x\right)$ with $e \notin\left\{e_{i}^{-1}, e_{(i+1)}(\bmod k)\right\}$, then we say that $e$ is right of $C$ if in the rotational order after $e_{(i+1)(\bmod k)}$, the edge $e$ comes before $e_{i}^{-1}$. Otherwise we say that $e$ is left of $C$. An undirected cycle $C$ corresponds to two directed cycles. If one of them is a face, we also call $C$ a facial cycle. Let $v_{i}, v_{i+1}, \ldots, v_{k}$ be a maximal subsequence of vertices of a directed cycle $C$ that all have no edges on the left (right) of $C$. If this sequence contains all vertices, then $C\left(C^{-1}\right)$ is a face. Otherwise (assume w.l.o.g. $i>0)$ we call $\left(e_{i-1}, e_{i}, \ldots, e_{k}\right)$ a left (right) facial subpath of $C$. In fact in that case $\left(e_{i-1}, e_{i}, \ldots, e_{k}\right)$, resp. $\left(e_{k}^{-1}, \ldots, e_{i-1}^{-1}\right)$ is part of a face. This concept is visualized in Figure 1.

Note that in a cubic graph the number of left and right facial subpaths of a directed cycle is 


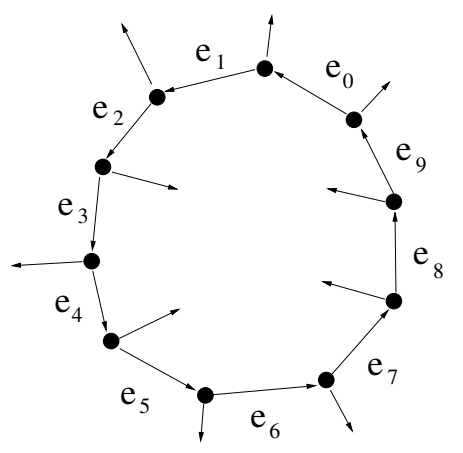

Fig. 1: A directed cycle as subgraph in a cubic embedded graph with left facial subpaths $\left(e_{9}, e_{0}, e_{1}, e_{2}\right)$, $\left(e_{3}, e_{4}\right),\left(e_{5}, e_{6}, e_{7}\right)$, and right facial subpaths $\left(e_{7}, e_{8}, e_{9}\right),\left(e_{2}, e_{3}\right)$, and $\left(e_{4}, e_{5}\right)$.

always the same and that the first and last edge of a left facial subpath always occur in right facial subpaths too (and the other way around). These are the edges with one endpoint with a left edge and one with a right edge. A cycle $C$ in a graph $G$ is called induced if all edges of $G$ with both endpoints on $C$ are also edges of $C$.

In the following we are especially interested in the genus of polyhedral embeddings. For an embedded graph, the genus is defined as the genus of the surface on which it is embedded. If $G$ is an abstract, not embedded, graph, then mingen $(G)$ is the smallest genus of a surface on which $G$ can be embedded. A genus embedding of a graph is an embedding in the smallest possible genus. Of course polyhedra - that is 3-connected plane graphs - all have a polyhedral embedding in the plane.

Due to Whitney's famous theorem, plane embeddings of 3-connected planar graphs are - up to mirror images - unique. In fact this is true in the more general setting of polyhedral embeddings, as due to Theorem 8.1 in Thomassen (1990) stating that embeddings of 3-connected planar graphs in genus at least 1 have facewidth at most 2 , and therefore cannot be polyhedral embeddings.

Theorem 1 Let $G$ be a 3-connected planar graph. Then the embedding in the plane is polyhedral and it is (up to mirror images) the unique plane embedding and also the unique polyhedral embedding.

This implies that there are no polyhedral embeddings of planar graphs, but genus embeddings of 3-connected planar graphs in the plane.

In Section 4 we will show that already for cubic graphs $G$ with $\operatorname{mingen}(G)=1$ even polyhedral embeddings with a genus close to the theoretical maximum for the number of vertices exist and that there are even cubic graphs that do have polyhedral embeddings, but not in minimum genus.

\section{Basic results}

Lemma 2 Let $P_{G}$ be a polyhedral embedding of a graph $G$ and $C=\left(v_{1}, v_{2}, \ldots, v_{k}\right)$ a simple induced directed cycle of $G$. Then $C\left(C^{-1}\right)$ either is a face or there are at least 3 vertices in $C$ that are starting points of an edge left (right) of $C$. If $G$ is cubic and neither $C$ nor $C^{-1}$ is a face, then $C$ has at least 2 left and at least 2 right facial subpaths. 


\section{Proof:}

Vertices with edges left (right) of $C$ separate $C$ into segments between two such vertices that belong to the same face. If $C\left(C^{-1}\right)$ is not a face and we have one vertex with edges to the left (right), then we have one segment and that would be part of a face with a facial walk that contains this vertex twice and is therefore not simple. If we have two such vertices, we have two segments. If they belong to different faces, we have two faces with the intersection containing these two vertices, which are not part of the same edge as $C$ is induced. Otherwise we have one cycle where these two vertices occur multiple times - so the facial walk would not be simple.

If $G$ is cubic and $C$ had exactly one left facial subpath, it also had one right facial subpath and these paths would share two edges, which is a contradiction to the embedding being polyhedral: if the subpaths are in the same face of the embedding, the face boundary would not be a cycle as two oppositely directed edges are contained and otherwise the two faces would share at least two edges.

A direct consequence of this lemma is the following corollary, which also contains the results from Lemma 2.1, Lemma 2.2 and Lemma 2.3(a) from Mohar and Vodopivec (2006):

Corollary 3 Let $P_{G}$ be a polyhedral embedding of a cubic graph $G$ and $C$ be an undirected cycle that is a 3-cycle, a 4-cycle, or an induced 5-cycle of $G$. Then-except if $G=K_{4}$ and $C$ is a 4-cycle $-C$ is a facial cycle.

If $C$ is an induced undirected 6-cycle, then $C$ is either a facial cycle or it has 3 edges to each side. Furthermore for both sets of three edges to one side, the starting vertices of the edges on $C$ do not form a path of length 2.

Note that if a 4-cycle in a cubic graph with more than 4 vertices is not induced, the graph has a 2-cut and therefore no polyhedral embedding in any surface.

\section{An algorithm to compute polyhedral embeddings of cubic graphs}

Already in Mohar and Vodopivec (2006) a computer program was used to check the existence of polyhedral embeddings of some cubic graphs. At that time Grünbaum's conjecture that there are no cubic graphs with chromatic index 4 that admit a polyhedral embedding in an orientable surface was still open and it was used to check all weak snarks on up to 30 vertices for the existence of a polyhedral embedding. We use the term weak snark for cyclically 4-connected cubic graphs with chromatic index 4 and - as usual - the term snark if in addition they have girth 5 . In Mohar and Vodopivec (2006) a straightforward algorithm was used that constructed all $2^{|V|-1}$ embeddings and tested them for being polyhedral. This approach is of course only suitable for relatively small lists of not too large graphs and would - even on a very large cluster - not be feasible for the graphs tested in this article, so that we had to develop a faster program, that we will refer to as poly_embed. In the meantime the conjecture has been refuted by Kochol Kochol (2009), but as for Kochol's counterexample it is neither known, whether it is smallest possible, nor whether it has smallest possible genus, we also tested the weak snarks on up to 36 vertices, which have been constructed in the meantime Brinkmann et al. (2013a) and are available at Brinkmann et al. (2013b). Furthermore we tested large lists of cubic graphs that are not snarks to find examples for the constructions in Section 4 
For a cubic graph $G$ we define two vertices of $G$ as being related, if they are contained in a common 3-, 4- or 5-cycle and begin by computing the equivalence classes of the transitive closure of this relation.

Proposition 4 If $v, w$ are vertices in the same equivalence class of a cubic graph $G$, then in any polyhedral embedding of $G$ the order of edges around $w$ is uniquely determined by the order around $v$.

Proof: For trivial equivalence classes the result is immediate, so assume that $f$ is a $3-$, 4- or 5 -cycle in $G$ containing the edges $\{x, v\}$ and $\{y, v\}$. Then fixing the rotation around $v$ fixes the order in which $f$ is traversed, w.l.o.g. $(v, x)=\operatorname{nx}((v, y))$. Due to Corollary 3 this implies that the rotation around $x$ is fixed, as the second edge incident with $x$ in the 5 -cycle must be the next in the rotational order after $(x, v)$. The rest of the proof follows by transitivity.

Note that for the transitive closure it makes no difference whether in the initial step you choose all 5-cycles or just the induced ones, as non-induced ones are the union of a 3- and a 4-cycle. Depending on the graph, there can be just one equivalence class in the best case, but if the girth is at least 6 , each vertex forms a separate equivalence class. Having computed the equivalence classes, we compute the set of compatible rotations. Already during this computation there can be conflicts implying that no polyhedral embeddings exist - see Figure 2 If in the recursion the rotation of a vertex in an equivalence class is switched, the rotations of all vertices in the class are switched simultaneously.

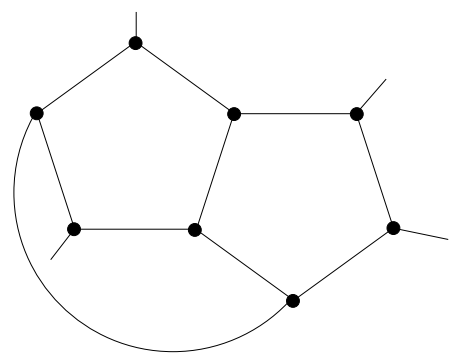

Fig. 2: An example of two 5-cycles and a 4-cycle that cannot be embedded as 5-gons and 4-gons.

The algorithm then starts by fixing the rotation of the largest equivalence class and assigning an initial rotation to all other classes. In the following branch and bound algorithm, the rotations of equivalence classes are recursively switched in order to enumerate all possible combinations. For graphs with girth 6 this does not help at all, as each equivalence class is just a single vertex, but even if the graph has just two 5-cycles sharing an edge, the number of possible rotations to be considered is already decreased by a factor of 128 . The recursion processes the equivalence classes in decreasing order of their size. Once a rotation is chosen, the rotation of the vertices in this class is considered to be fixed until the recursion returns to this class. So at each recursion depth we have a set of fixed vertices. As their rotation will not be modified at higher depths of 
the recursion, a contradiction within this set - e.g. a face with only fixed vertices and without a simple cycle as boundary - allows to bound the recursion and backtrack.

We use the following observation when testing for polyhedral embeddings. It is based on the fact that in a cubic graph two cycles sharing a vertex share a whole edge.

Note 1 An embedding of a cubic graph is polyhedral if and only if

- no vertex occurs twice in a facial walk and

- no two faces share more than two vertices.

The advantage of this characterization from a computational point of view is that the second criterion, which is a criterion about all pairs of faces, is reduced to a very simple test. If the faces are implemented as bitvectors, it is sufficient to compute the intersection - a very simple and fast operation for sets represented as bitvectors - and afterwards test whether the intersection contains more than two bits - again very fast. For graphs that are not cubic, an extra test would be needed for the case of two common vertices in order to test whether they are the endpoints of a common edge lying in both faces.

During the recursion, the algorithm distinguishes between the case when the next equivalence class is a single vertex or not. As long as there are nontrivial equivalence classes, that are not fixed, they are processed in decreasing order of their size. When there are only trivial equivalence classes left, we use the concept of obstruction: an obstruction in an embedded graph is a part of the boundary between two occurences of the same vertex or the union of the vertex sets of two faces which share at least 3 vertices.

For trivial equivalence classes, at each recursion depth, a set $S=v_{1}, v_{2}, \ldots, v_{k}$ is chosen and for each $1 \leq i \leq k$ the vertices $v_{1}, \ldots, v_{i-1}$ are declared fixed (with the rotation they initially have) and vertex $v_{i}$ is declared fixed after the rotation was switched. For each $i$ then the next recursion depth is called with the vertices $v_{i+1}, \ldots, v_{k}$ being not fixed and available for the next set. If there is no obstruction, the embedding is polyhedral and output (or counted if the options require no output) and $S$ is chosen as the set of all vertices that are not yet fixed. Otherwise $S$ is chosen as the set of not fixed vertices in an obstruction.

After each switching of a rotation, Corollary 3 is applied to all hexagons. This might lead to a contradiction or to uniquely determined rotations for some not yet fixed vertices in these hexagons. If no contradiction is found, a - preferably small - obstruction is searched. If there is none, the embedding is polyhedral and if there is one with only fixed vertices, we can backtrack.

In fact it turned out to be faster not to do a complete search for obstructions with only fixed vertices - except at the end of the recursion or very close to the root of the recursion.

Testing the program: In order to test the program we wrote two other programs. A first program creates all possible embeddings of a given graph simply by writing all possible combinations of orders around each vertex. We then independently wrote a program to check whether a given embedded graph is polyhedral by checking that each face is a cycle and checking the intersection of each pair of faces. We compared the number of polyhedral embeddings and their genus for all cubic graphs on up to 22 vertices. For 24 vertices only the cubic graphs with girth at least 4 were independently tested. We had agreement in all cases. 
Performance: All times are given on a linux laptop with Core i7-6820HQ CPU, $2.70 \mathrm{GHz}$ (running typically at $3.2 \mathrm{Ghz}$ ). Constructing all polyhedral embeddings of all 117,940,535 cubic graphs on 24 vertices takes 14 minutes, so in average 140,000 graphs per second are processed. The speed is for a large part due to the presence of small cycles exploited in the first step of the algorithm. Restricting the tests to the cubic graphs on 24 vertices with girth at least 6 , it takes 6.4 seconds for 7,574 graphs - so only around 1,200 per second. Testing all 153,863 weak snarks on up to 30 vertices takes about 0.55 seconds. Testing all 432,105,682 weak snarks on up to 36 vertices takes a bit less than half an hour. Constructing all polyhedral embeddings of the Kochol snark on 74 vertices takes 0.03 seconds. Note that the Kochol snark has many pentagons.

\subsection{Computational results}

In Kochol (2009) Kochol refuted Grünbaum's conjecture that cubic graphs that have a polyhedral embedding also have an edge-3-colouring Grünbaum (1969), by constructing a snark with 74 vertices and a polyhedral embedding. It has genus 5 and the (unique) genus embedding is polyhedral and in fact also the only polyhedral embedding. The fact that the genus embedding is unique is - just like all other genera in this paper - computed by the program multi_genus described in Brinkmann (2020). It is neither known whether Kochol's graph is the smallest counterexample nor whether there are counterexamples with smaller genus. So we tested all available smaller snarks and weak snarks in the database HoG Brinkmann et al. (2013b), that is all weak snarks on up to 36 vertices, an incomplete list of $19,775,768$ snarks on 38 vertices and 25 snarks with girth 6 on 40 vertices. None of them allows a polyhedral embedding.

Of course snarks are non-hamiltonian, but having no hamiltonian cycle is a much weaker condition than being a snark, so a question is when the first non-hamiltonian cubic graphs with a polyhedral embedding occur. The smallest such graph is the Coxeter graph, which has 28 vertices. It is in fact the only cubic nonhamiltonian graph on up to 28 vertices with a polyhedral embedding. The Coxeter graph has genus 3 and 16 embeddings in genus 3, which are all isomorphic. The embeddings in genus 3 are the only polyhedral ones.

In order to get an idea of how common polyhedral embeddings of cubic graphs are, but also in order to search for small examples of cubic graphs with several polyhedral embeddings, polyhedral embeddings in more than one genus and even graphs with polyhedral embeddings, but not in their minimum genus, we tested all cubic graphs on up to 28 vertices for polyhedral embeddings. The lists of graphs were generated by the program minibaum described in Brinkmann (1996). The results are given in Table 1 and some special embeddings found are given and used for the construction of infinite sequences in Section 4 .

In Table 2 numbers of non-isomorphic polyhedrally embedded cubic graphs are given. They were obtained by filtering the output of poly_embed for non-isomorphic embeddings and computing the genus of the underlying graphs by multi_genus. Although even for 28 vertices the ratio of polyhedral embeddings that are not in minimum genus is still only $0.00016 \%$, in the last step it grows much faster than the number of polyhedral embeddings. The fact that the same graph embedded in a small genus has more, and in average smaller, faces than when embedded in a larger genus, suggests that polyhedral embeddings should more often occur in or close to the smallest genus that the graph can be embedded in. Though the computed numbers seem to 


\begin{tabular}{l|c|c|c|c}
$\begin{array}{c}\text { number } \\
\text { of } \\
\text { vertices }\end{array}$ & $\begin{array}{c}\text { number } \\
\text { of graphs }\end{array}$ & $\begin{array}{c}\text { graphs } \\
\text { with pol. emb. }\end{array}$ & $\begin{array}{c}\text { graphs with } \\
\text { more than } \\
\text { one pol. emb. }\end{array}$ & $\begin{array}{c}\text { graphs with pol. } \\
\text { emb. in more } \\
\text { than one genus }\end{array}$ \\
\hline 4 & 1 & $1(100 \%)$ & 0 & 0 \\
6 & 2 & $1(50 \%)$ & 0 & 0 \\
8 & 5 & $2(40 \%)$ & 0 & 0 \\
10 & 19 & $5(26 \%)$ & 0 & 0 \\
12 & 85 & $14(16.5 \%)$ & 0 & 0 \\
14 & 509 & $51(10 \%)$ & 1 & 0 \\
16 & 4,060 & $240(5.9 \%)$ & 4 & 0 \\
18 & 41,301 & $1,349(3.3 \%)$ & 28 & 0 \\
20 & 510,489 & $9,464(1.9 \%)$ & 278 & 0 \\
22 & $7,319,447$ & $84,230(1.2 \%)$ & 2,997 & 4 \\
24 & $117,940,535$ & $909,431(0.77 \%)$ & 32,438 & 157 \\
26 & $2,094,480,864$ & $10,902,162(0.52 \%)$ & 348,078 & 0 \\
28 & $40,497,138,011$ & $138,008,652(0.34 \%)$ & $3,909,031$ &
\end{tabular}

Tab. 1: Computational results on all cubic graphs with a given number of vertices. Note that "with more than one polyhedral embedding" refers to different combinatorial embeddings in the sense of being not identical or mirror images. It does not necessarily refer to non-isomorphic embeddings.

support such a claim, the numbers are much too small to draw well grounded conclusions.

\section{Constructions and further results on polyhedral embeddings}

As Theorem 1 states, polyhedral embeddings of 3-connected planar graphs are unique and always in the plane - which is of course also the minimum genus in which the graph can be embedded. This behaviour changes dramatically once we move away from planar graphs. Already for genus 1 there are cubic graphs $G$ with mingen $(G)=1$ that have polyhedral embeddings of different genera as well as such graphs with polyhedral embeddings - but not in minimum genus.

As the number of faces decreases when the genus increases, there is a natural upper bound for the genus of a polyhedral embedding with a given number of vertices and edges:

Lemma 5 If $P$ is a polyhedral embedding of a cubic graph with $n$ vertices, then the genus $g(P)$ fulfills

$$
g(P) \leq \frac{n-\sqrt{12 n+1}+3}{4}
$$

This bound is sharp for infinitely many $P$. To be exact: for each $n$ for which the right hand side of the formula is an integer number, there is a polyhedral embedding $P$ of a cubic graph with $n$ vertices and genus $g(P)=\frac{n-\sqrt{12 n+1}+3}{4}$.

\section{Proof:}

An embedding of a cubic graph is polyhedral if and only if the dual is a simple graph. As the dual graph also has $\frac{3 n}{2}$ edges and a simple graph with $f$ vertices has at most $\frac{f(f-1)}{2}$ edges, we get 
On the genera of polyhedral embeddings of cubic graphs

\begin{tabular}{l|c|c|c}
$\begin{array}{c}\text { number } \\
\text { of } \\
\text { vertices }\end{array}$ & $\begin{array}{c}\text { number } \\
\text { of pol. } \\
\text { embeddings }\end{array}$ & $\begin{array}{c}\text { number of } \\
\text { min genus } \\
\text { pol. emb. }\end{array}$ & $\begin{array}{c}\text { number of } \\
\text { not min genus } \\
\text { pol. emb. }\end{array}$ \\
\hline 4 & 1 & 1 & 0 \\
6 & 1 & 1 & 0 \\
8 & 2 & 2 & 0 \\
10 & 5 & 5 & 0 \\
12 & 14 & 14 & 0 \\
14 & 51 & 51 & 0 \\
16 & 240 & 240 & 0 \\
18 & 1,361 & 1,361 & 0 \\
20 & 9,704 & 9,704 & 0 \\
22 & 87,433 & 87,433 & 0 \\
24 & 946,083 & 946,083 & 0 \\
26 & $11,298,676$ & $11,298,671$ & 5 \\
28 & $142,414,959$ & $142,414,731$ & 228
\end{tabular}

Tab. 2: Computational results on all non-isomorphic polyhedrally embedded cubic graphs with a given number of vertices. Here, non-isomorphic refers to the concept of isomorphism of embedded graphs - not of abstract graphs.

$f \geq \sqrt{3 n+0.25}+0.5$ and inserting this into the Euler formula and the formula for the genus we get the result in the lemma.

If $\frac{n}{4}-\frac{\sqrt{3 n+0.25}}{2}+\frac{3}{4}$ is an integer number, replacing $n$ by $\frac{f(f-1)}{3}$ and simplifying the term gives that this is the case if and only if $\frac{(f-3)(f-4)}{12}$ is an integer. This can easily be seen to be the case if and only if $f(\bmod 12) \in\{0,3,4,7\}-$ allowing a triangular embedding of the complete graph $K_{f}$ (see Gross and Tucker (1987), Ringel and Youngs (1968), or Sun (2021)) with the given genus, which has a simple cubic dual with the given number $n$ of vertices.

Note that the dual cubic graph of a triangular embedding of a complete graph is polyhedrally embedded in the same genus as the complete graph, but that this is not necessarily a minimum genus embedding. In fact tests for duals of some embeddings of $K_{12}$ gave cubic graphs with 44 vertices and genus 4 or 5 (instead of 6 as $K_{12}$ ), but the only polyhedral embeddings were in genus 6. We did not do a complete enumeration of all possible embeddings of $K_{12}$.

In Theorem 8.2 of Thomassen (1990) an infinite sequence of toroidal cubic graphs is given that - with $n$ the number of vertices - have an embedding with facewidth at least 4 in genus $\frac{n}{8}-2 \sqrt{\frac{n}{8}}+1$, so up to minor terms about half the genus of the maximum possible genus. If one only requires that the embedding is polyhedral, we can - up to minor terms - even get the same value as the maximum possible genus. We first need the following lemma:

Lemma 6 Let $G$ be the graph of a hexagonal tiling $T$ of the torus where all cycles of length at most 6 are faces. Then $G$ has (up to mirror images) at most 2 polyhedral embeddings.

\section{Proof:}


The toroidal embedding $T$, its mirror image $T^{-1}$, as well as the two embeddings $T_{a}, T_{a}^{-1}$ (one a mirror image of the other) where in each hexagon the rotations are alternatingly like in $T$ and like in $T^{-1}$ might be polyhedral. Note that if in $T_{a}$ or $T_{a}^{-1}$ the rotations in one original hexagon are given, they are uniquely determined for all others.

If $T^{\prime}$ is a polyhedral embedding of $G$ that is none of these, then - due to Corollary 3 - there is a hexagon where at three vertices the rotation is like in $T$ and at three vertices the rotation is like in $T^{-1}$ and as $T^{\prime}$ is neither $T_{a}$ nor $T_{a}^{-1}$, the hexagon can also be chosen in a way that at two neighbouring vertices the rotation is like in $T^{-1}$. In Figure 3 the two possible distributions of rotations in $T^{\prime}$ for the two hexagons of $T$ sharing this edge are depicted. Parts of two faces of $T^{\prime}$ are displayed by bold resp. dotted lines. As these two faces share at least two edges, $T^{\prime}$ cannot be a polyhedral embedding. The condition that there are no short non-facial cycles is needed to guarantee that the two edges in Figure 3 leading to the contradiction are distinct.

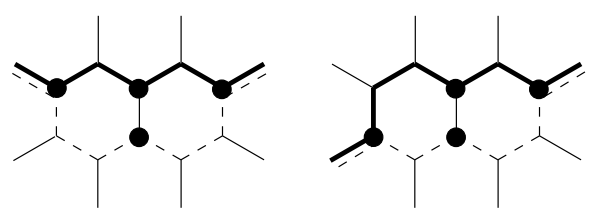

Fig. 3: Portions of the hexagonal embeddings $T$ and $T^{\prime}$ on the torus. $T$ is described by choosing the rotation as given by the normal clockwise traversal of edges around all vertices. For $T^{\prime}$ the rotation is reversed - that is: like in $T^{-1}$ - for bold vertices and like in $T$ for the other vertices. Parts of two faces of $T^{\prime}$ are marked with bold resp. dotted lines.

Lemma 7 There are infinitely many $n$, so that there is a cubic graph with $n$ vertices and with a polyhedral embedding on the torus as well as on a surface of genus $\frac{n}{4}-\frac{3}{2} \sqrt{\frac{n}{2}}+1$ - and no other polyhedral embedding.

\section{Proof:}

Let $n$ be any number, so that $n=2 k^{2}$ for some $k \geq 3$. Let $T$ be the cubic bipartite polyhedral embedding on the torus, formed by a $k \times k$ parallelogram of $k^{2}$ hexagons, depicted for $k=4$ in Figure 4 with the sides identified like indicated by the letters in the figure. This cubic graph has $n=2 k^{2}$ vertices and $3 k^{2}$ edges. If we switch the rotation at each vertex of one of the bipartition classes, the faces of this new graph are zig-zag walks in the old one, also known as Petrie walks in a more general context. They are depicted in bold in Figure 4 for three closed Petrie walks. Each face is a simple cycle and has $2 k$ edges. As each edge is in two faces this gives $3 k$ faces and therefore a genus of $\frac{k^{2}-3 k+2}{2}$. Replacing $k$ by $\sqrt{\frac{n}{2}}$, we get the result in the lemma. It is easy to see that two faces either share no edges - if they correspond to parallel Petrie walks - or one edge, so that the embedding is polyhedral.

The last part is a direct consequence of Lemma 6 and that for $k=3$ (so $n=18$ ) - where Lemma 6 cannot be applied - we get $\frac{n}{4}-\frac{3}{2} \sqrt{\frac{n}{2}}+1=1$. 


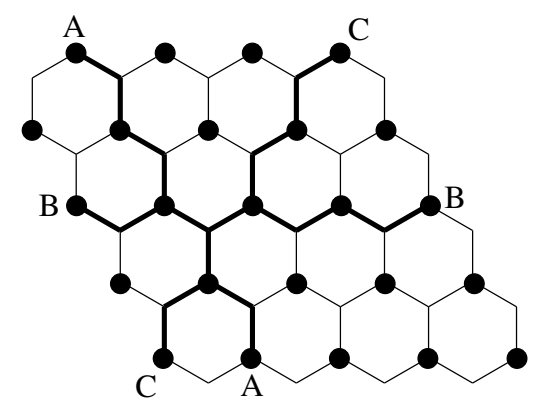

Fig. 4: Two embeddings of a cubic graph: a hexagonal embedding $T$ on the torus with 16 hexagons is given by the rotation defined by the clockwise traversal of edges around all vertices. A second embedding is described by defining the rotation around bold vertices to be reversed (that is: coming from the counterclockwise traversal of the edges) and around the other vertices like in $T$. Some facial cycles of the second embedding, which is in a higher genus are marked with fat lines.

For a cubic graph $G$ and a genus $g$ let $n_{g}(G)$ denote the number of different polyhedral embeddings of $G$ in genus $g$ with mirror images identified (or equivalently: the rotation around one vertex fixed). When referring to different polyhedral embeddings, we always assume that mirror images are considered to represent the same embedding.

Note that the Heawood graph $H$ on the torus - that is: the dual of $K_{7}$ embedded on the torus has many 6-cycles that are not faces. It is a cubic hexagonal tiling and has (up to mirror images) 8 different polyhedral embeddings on the torus, so $n_{1}(H)=8$. These 8 embeddings are isomorphic. We will now prove the existence of some cubic graphs with special embedding properties:

Theorem 8 For each $k>0$ there is

(a) a cubic graph $G$ admitting two polyhedral embeddings $P, P^{\prime}$ so that the difference between the genera of these embeddings is at least $k$ and there is no embedding for any genus in between.

(b) a cubic graph $G$ admitting polyhedral embeddings in at least $k$ genera.

(c) a cubic graph $G$ and a genus $g$, so that $n_{g}(G) \geq k$. In fact for genus $g$ there is a graph $G$ with $n_{g}(G) \geq 8^{g}$. This is also true if one requires the embeddings to be non-isomorphic.

(d) a cubic graph $G$, so that the smallest genus of a polyhedral embedding of $G$ differs from mingen $(G)$ by at least $k$.

In fact (a) is a direct consequence of Lemma 7 and mentioned here only for completeness. In order to prove the other items, we will now first present an operation on graphs that preserves some properties with respect to polyhedral embeddings. Then we will present some graphs with special properties of their polyhedral embeddings, which we will use as building blocks for this operation. 


\subsection{Combining graphs}

The following construction for nontrivial 3-cuts has already been used in several papers under various names and to study various invariants. It was e.g. called marriage in Kotzig (1964) when studying Hamiltonian cycles or star product in Mohar and Vodopivec (2006) when studying edge colourings of cubic graphs in the context of polyhedral embeddings.

For a graph $G$ with a vertex $v$ let $G_{v}$ denote the graph $G$ with the vertex $v$ deleted.

Let $G, G^{\prime}$ be graphs with cubic vertices $v$ in $G$ and $v^{\prime}$ in $G^{\prime}$ and let the neighbours of $v$ be $a, b, c$ and those of $v^{\prime}$ be $a^{\prime}, b^{\prime}, c^{\prime}$. Then $G_{v} \star_{\left(a, b, c, a^{\prime}, b^{\prime}, c^{\prime}\right)} G_{v^{\prime}}^{\prime}$ denotes a graph obtained by connecting $G_{v^{\prime}}^{\prime}$ to $G_{v}$ by adding the edges $\left\{a, a^{\prime}\right\},\left\{b, b^{\prime}\right\},\left\{c, c^{\prime}\right\}$. If $G, G^{\prime}$ are embedded, the edges are inserted in the rotation at the same place where the edges from $v$, resp. $v^{\prime}$ were located. In cases where the exact identification is not important or clear from the context, we also write shortly $G_{v} \star G_{v^{\prime}}^{\prime}$ to simplify notation. If $G, G^{\prime}$ are embedded with genus $g$, resp. $g^{\prime}$, in $G_{v}$ the vertices $a, b, c$ occur in the order $a, b, c$ and in $G_{v^{\prime}}^{\prime}$ the vertices $a^{\prime}, b^{\prime}, c^{\prime}$ occur in the order $a^{\prime}, c^{\prime}, b^{\prime}$, the fact that $G_{v} \star_{\left(a, b, c, a^{\prime}, b^{\prime}, c^{\prime}\right)} G_{v^{\prime}}^{\prime}$ is embedded with genus $g+g^{\prime}$ can now be obtained by the fact that (with $v()$ the number of vertices, $e()$ the number of edges and $f()$ the number of faces of a graph) $v\left(G_{v} \star G_{v^{\prime}}^{\prime}\right)=v(G)+v\left(G^{\prime}\right)-2, e\left(G_{v} \star G_{v^{\prime}}^{\prime}\right)=e(G)+e\left(G^{\prime}\right)-3$, and $f\left(G_{v} \star G_{v^{\prime}}^{\prime}\right)=f(G)+f\left(G^{\prime}\right)-3$. As minimum genus embeddings of $G$ and $G^{\prime}$ can be chosen in a way that the rotations around $v$ and $v^{\prime}$ imply this order, we get mingen $\left(G_{v} \star G_{v^{\prime}}^{\prime}\right) \leq \operatorname{mingen}(G)+\operatorname{mingen}\left(G^{\prime}\right)$ for all possible $\left(a, b, c, a^{\prime}, b^{\prime}, c^{\prime}\right)$.

Lemma 10 is essentially Theorem 3.1 from Mohar and Vodopivec (2006), but as we need it in a slightly different formulation and also allow non-cubic graphs, we will just reprove it here:

We will need the following proposition also following from the proofs of Theorem 3.1, Theorem 3.3 and Theorem 3.5 in Mohar and Vodopivec (2006). An edge cut $S$ is a set of edges, so that there exists a bipartition of the vertex set of the graph, so that $S$ is the set of edges with endpoints in both sets.

Proposition 9 For $3 \leq k \leq 5$ let $e_{1}, e_{2}, \ldots, e_{k}$ be a $k$-edge-cut in a polyhedral embedding of a (not necessarily cubic) graph $G$. Then the subgraph of the dual graph whose vertices are the faces containing an edge in $\left\{e_{1}, e_{2}, \ldots, e_{k}\right\}$ and whose edges correspond to $e_{1}, e_{2}, \ldots, e_{k}$ is a cycle. Furthermore: if $C$ is a component of $G-\left\{e_{1}, e_{2}, \ldots, e_{k}\right\}$ with the embedding induced by $G$, then all endpoints of $e_{1}, e_{2}, \ldots, e_{k}$ in $C$ are in the same face of $C$.

\section{Proof:}

Each of the faces $f_{1}, \ldots, f_{j}$ containing at least one of $e_{1}, e_{2}, \ldots, e_{k}$ contains an even number of these edges, as otherwise an edge has two endpoints in the same bipartition class. Let $D$ be the graph with vertices $f_{1}, \ldots, f_{j}$ and edges $\left\{f_{i}, f_{h}\right\}$ for all $f_{i}, f_{h}$ that share one of $e_{1}, e_{2}, \ldots, e_{k}$. Due to polyhedrality $D$ is a simple graph. As there are at most 5 edges, we have immediately that the vertex degrees in $D$ are only 2 or 4 and it is easy to see that vertices with degree 4 cannot exist. So $D$ is a simple cycle (so also $j=k$ ).

This means that each $f_{i} \in\left\{f_{1}, \ldots, f_{k}\right\}$ has in each of the two components containing vertices of $f_{i}$ exactly one facial subpath or only a single vertex and the union of subpaths in the same component forms a closed facial walk after the removal of $e_{1}, \ldots, e_{k}$. 

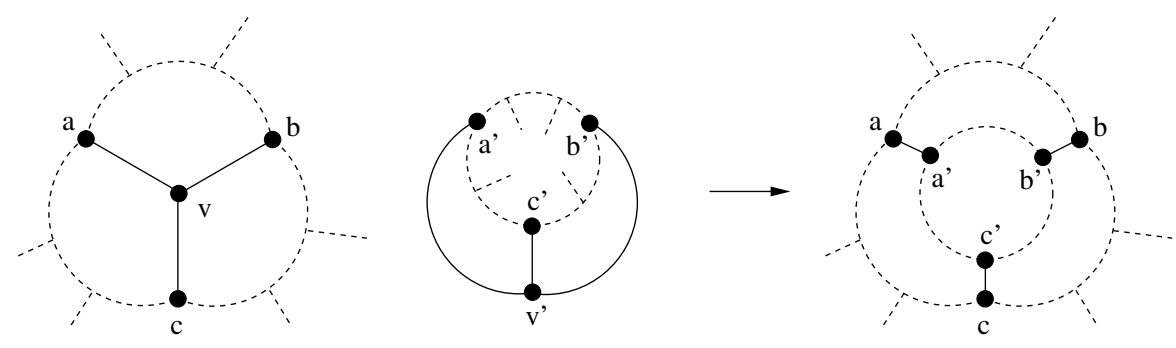

Fig. 5: The operation $G_{v} \star G_{v^{\prime}}^{\prime}$.

Lemma 10 Let $G$ be a graph with a polyhedral embedding in genus $g$ and a nontrivial 3-edge-cut.

Then there are (smaller) embedded graphs $G^{\prime}, G^{\prime \prime}$ with cubic vertices $v^{\prime}$ in $G^{\prime}$ and $v^{\prime \prime}$ in $G^{\prime \prime}$ and polyhedral embeddings in genus $g^{\prime}$, resp. $g^{\prime \prime}$, so that $g^{\prime}+g^{\prime \prime}=g$ and $G=G_{v^{\prime}}^{\prime} \star G_{v^{\prime \prime}}^{\prime \prime}$. If $G$ is cubic, then also $G^{\prime}$ and $G^{\prime \prime}$ are cubic.

Proof: As $G$ is 3-edge-connected, after the removal of the edge cut we have exactly two (embedded) components and due to Proposition 9 both have the three distinct (as $G$ is 3-connected) vertices where an edge was removed in the same face. So we can add vertices $v^{\prime}$, resp. $v^{\prime \prime}$ inside the face to form $G^{\prime}$ and $G^{\prime \prime}$. The fact that $g^{\prime}+g^{\prime \prime}=g$ can again be shown by simply counting vertices, edges and faces and the fact that $G^{\prime}$ and $G^{\prime \prime}$ are polyhedrally embedded can be easily seen by checking the face adjacencies around the new vertices.

Lemma 11 Let $G, G^{\prime}$ be graphs with cubic vertices $v$ in $G$ and $v^{\prime}$ in $G^{\prime}$.

Then for any genus $g$ we have with $0 \leq j, j^{\prime} \leq g$

$$
n_{g}\left(G_{v} \star G_{v^{\prime}}^{\prime}\right)=\sum_{j+j^{\prime}=g}\left(n_{j}(G) \cdot n_{j^{\prime}}\left(G^{\prime}\right)\right) .
$$

\section{Proof:}

Let $a, b, c$, resp. $a^{\prime}, b^{\prime}, c^{\prime}$ be the neighbours of $v$ in $G$, resp. $v^{\prime}$ in $G_{v^{\prime}}^{\prime}$, named in a way that the edges $\left\{a, a^{\prime}\right\},\left\{b, b^{\prime}\right\}$ and $\left\{c, c^{\prime}\right\}$ are inserted when forming $G_{v} \star G_{v^{\prime}}^{\prime}$.

Let now $j, j^{\prime}$ be so that $0 \leq j, j^{\prime} \leq g$ and $j+j^{\prime}=g$. Furthermore let $I_{G}$ be a polyhedral embedding of $G$ of genus $j$ with the rotation around $v$ equal to $a, b, c$ and let $I_{G^{\prime}}$ be a polyhedral embedding of $G^{\prime}$ of genus $j^{\prime}$ with the rotation around $v^{\prime}$ equal to $a^{\prime}, c^{\prime}, b^{\prime}$. Inserting the edges $\left\{a, a^{\prime}\right\},\left\{b, b^{\prime}\right\}$ and $\left\{c, c^{\prime}\right\}$ in the rotational order around $a, b, c, a^{\prime}, b^{\prime}, c^{\prime}$ where the deleted edges in $I_{G}$ and $I_{G^{\prime}}$ have been - see Figure 5 - we get an embedding of $G_{v} \star G_{v^{\prime}}^{\prime}$ with genus $j+j^{\prime}=g$ and by checking face adjacencies it is easily seen to be polyhedral.

This way all polyhedral embeddings of $G$ with genus $j$ can be combined with all polyhedral embeddings of $G^{\prime}$ with genus $j^{\prime}$ and doing this for all possible $j, j^{\prime}$ we get $n_{g}\left(G_{v} \star G_{v^{\prime}}^{\prime}\right) \geq$ $\sum_{j+j^{\prime}=g}\left(n_{j}(G) \cdot n_{j^{\prime}}\left(G^{\prime}\right)\right.$ ). Note that two different embeddings for $G$ (and analogously $G^{\prime}$ ) lead to different embeddings of $G_{v} \star G_{v^{\prime}}^{\prime}$, as the rotation around $v$ is always the same, so the difference is at vertices also present in $G_{v} \star G_{v^{\prime}}^{\prime}$. 

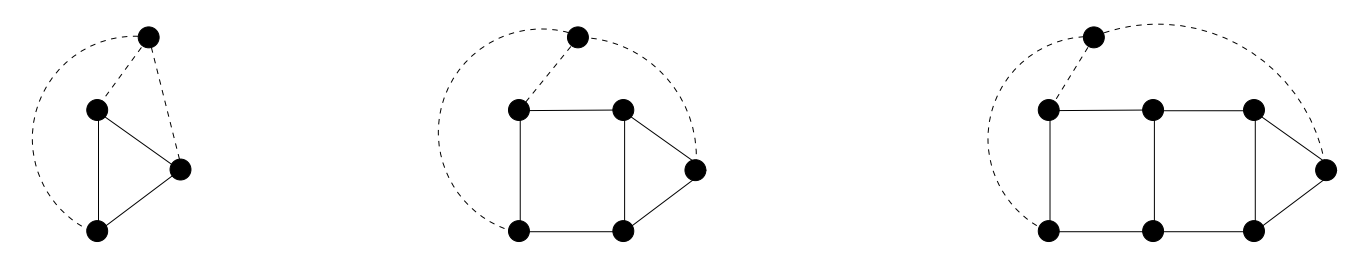

Fig. 6: 3-connected plane graphs, so that after the removal of a vertex only pairwise non-isomorphic graphs with only triangles and quadrangles as bounded faces remain.

What remains to be shown is that each polyhedral embedding of $G_{v} \star G_{v^{\prime}}^{\prime}$ is of this form. Assume a polyhedral embedding of genus $g$ of $G_{v} \star G_{v^{\prime}}^{\prime}$ to be given. Choose that of the two equivalent embeddings where in the face containing the edges $\left\{a, a^{\prime}\right\},\left\{b, b^{\prime}\right\}$ the facial walk is $b^{\prime}, b, \ldots, a, a^{\prime}, \ldots$ (in the mirror image it is $b, b^{\prime}, \ldots, a^{\prime}, a, \ldots$ ). Then in the face containing $\left\{b, b^{\prime}\right\},\left\{c, c^{\prime}\right\}$ the facial walk is $c^{\prime}, c, \ldots, b, b^{\prime}, \ldots$ and we get that in the component containing $a, b, c$ the order in the face is $a, c, b$, so that if we insert a vertex into the face and connect it to the three vertices by assigning the reverse order, we get an embedding of $G$ with the order around the new vertex $a, b, c$. Analogously we get an embedding of $G^{\prime}$ with rotational order around the new vertex $a^{\prime}, c^{\prime}, b^{\prime}$, so that the embedding is in fact of the form counted in the formula.

The following corollary is a direct consequence of Lemma 11 and Theorem 1 , which implies that for 3 -connected planar graphs $G^{\prime}$ we have $n_{0}\left(G^{\prime}\right)=1$ and $n_{j^{\prime}}\left(G^{\prime}\right)=0$ for $j^{\prime}>0$.

Corollary 12 Let $G, G^{\prime}$ be graphs with cubic vertices $v$ in $G$ and $v^{\prime}$ in $G^{\prime}$ and let $G^{\prime}$ be a 3-connected planar graph.

Then for all $g$ we have $n_{g}\left(G_{v} \star G_{v^{\prime}}^{\prime}\right)=n_{g}(G)$.

Lemma 13 Let $G$ be a cubic graph with $k$ different (isomorphic or non-isomorphic) polyhedral embeddings of some genus $g$. Then there is a graph $G^{\prime}$ that has $k$ different non-isomorphic polyhedral embeddings of genus $g$

\section{Proof:}

If the vertices of $G$ are $w_{1}, \ldots, w_{n}$, we can choose $n 3$-connected plane cubic graphs $G_{1}, \ldots, G_{n}$ and vertices $v_{1}, \ldots, v_{n}$, so that for $1 \leq i \leq n, v_{i}$ is a vertex of $G_{i}$ and the graphs $\left(G_{i}\right)_{v_{i}}$ are pairwise non-isomorphic graphs with only triangles and quadrangles as bounded faces (see e.g. Figure 6). Now we can iteratively apply the star operation to replace each $w_{i}$ by $\left(G_{i}\right)_{v_{i}}$ to form a graph $G^{\prime}$. According to Corollary 12 G $G^{\prime}$ also has $k$ polyhedral embeddings on genus $g$. As all faces in one of these embeddings that are not contained in a subgraph $\left(G_{i}\right)_{v_{i}}$ are at least hexagons, each isomorphism between two such embeddings must stabilize each subgraph as well as the set of original edges - that is edges not in one of the $\left(G_{i}\right)_{v_{i}}$. As the subgraphs $\left(G_{i}\right)_{v_{i}} 1 \leq i \leq n$ are equivalence classes in the sense of Proposition 4 there are just two possible rotations inside the subgraphs and an embedding isomorphism between two different embeddings $I_{1}, I_{2}$ must therefore have an original edge with endpoints in subgraph $\left(G_{i}\right)_{v_{i}}$ and $\left(G_{j}\right)_{v_{j}}$ where at the vertex in $\left(G_{i}\right)_{v_{i}}$ the rotation is as in $I_{1}$ and at the vertex in $\left(G_{j}\right)_{v_{j}}$ it is different from the one in $I_{1}$. This means that the embedding isomorphism interchanges the other two subgraphs sharing an original edge 
with $\left(G_{j}\right)_{v_{j}}$ compared to $I_{1}$, which is impossible as the subgraphs are stabilized. So there is no embedding isomorphism mapping $I_{1}$ to $I_{2}$.

The smallest cubic graph with 8 different (but isomorphic) embeddings on the torus is the Heawood graph. The construction in the proof of Lemma 13 with replacing all vertices is of course designed for simplicity and not for a minimal number of vertices. Nevertheless a smallest cubic graph with 8 non-isomorphic embeddings on the torus - which has 22 vertices - can be obtained from the Heawood graph by applying the star operation.

Lemma 11 now has the following direct consequences:

\section{Corollary 14}

(a) If there is a cubic graph $G$ with mingen $(G)=g$ and the smallest genus of a polyhedral embedding is $g^{\prime}$, then there is also a cubic graph with genus at most $2 g$ and the smallest genus of a polyhedral embedding is $2 g^{\prime}$.

(b) If there is a cubic graph $G$ with polyhedral embeddings in genus $g_{1}<g_{2}<\cdots<g_{m}$, and a cubic graph $G^{\prime}$ with polyhedral embeddings in genus $g_{1}^{\prime}<g_{2}^{\prime}<\cdots<g_{k}^{\prime}$, then there is also a cubic graph, so that for all $1 \leq i \leq m$ and $1 \leq j \leq k$ there is a polyhedral embedding in genus $g_{i}+g_{j}^{\prime}$.

(c) If there is a cubic graph $G$ with $p$ (up to mirror images) different polyhedral embeddings in genus $g$ and a cubic graph $G^{\prime}$ with $p^{\prime}$ (up to mirror images) different polyhedral embeddings in genus $g^{\prime}$, then there is a cubic graph with $p \cdot p^{\prime}$ different polyhedral embeddings in genus $g+g^{\prime}$.

\section{Proof:}

Part (a) is a consequence of the formula in Lemma 11 applied to $G_{v} \star G_{v}$ for an arbitrary vertex $v$ in $G$. By Lemma 11 the smallest possible polyhedral embedding of $G_{v} \star G_{v}$ is in genus $2 g^{\prime}$ while $\operatorname{mingen}\left(G_{v} \star G_{v}\right) \leq 2$ mingen $(G)$.

Part (b) and (c) are again a direct consequence of the formula in Lemma 11 applied to $G_{v} \star G_{v^{\prime}}^{\prime}$ for arbitrary vertices $v$ in $G$ and $v^{\prime}$ in $G^{\prime}$.

So in order to prove Theorem 8, we just need some graphs that can be used as initial graphs for Corollary 14.

\subsection{Some special graphs}

We will write $G \star G^{\prime}$ as a notation for a graph where arbitrary vertices $v$ in $G$ and $v^{\prime}$ in $G^{\prime}$ can be taken to form $G_{v} \star G_{v^{\prime}}^{\prime}$. The Heawood graph $H$ has 8 different (but isomorphic) embeddings on the torus. With Corollary 14 (c) this implies that for each $g$ the graph $(\ldots(H \star H) \star H) \star \cdots \star H)$ where the star operation is applied $g$ times has a total of $8^{g}$ polyhedral embeddings in genus $g$, which implies the first two parts of Theorem 8 (c). The last part follows with Lemma 13. 


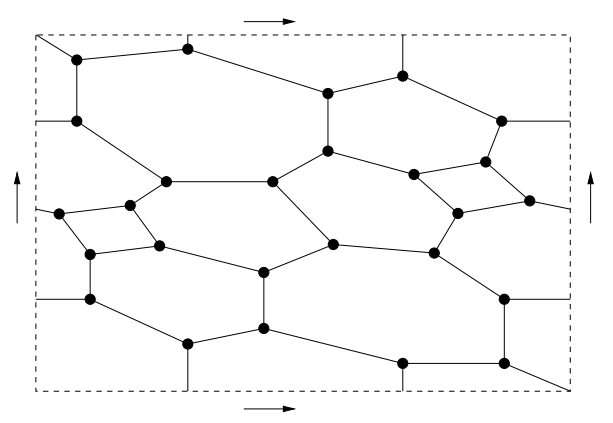

Fig. 7: The polyhedral embedding of genus 1 for one of the smallest cubic graphs on 26 vertices that have polyhedral embeddings of different genera. As usual, opposite sides of the rectangle need to be identified as specified by the arrows.
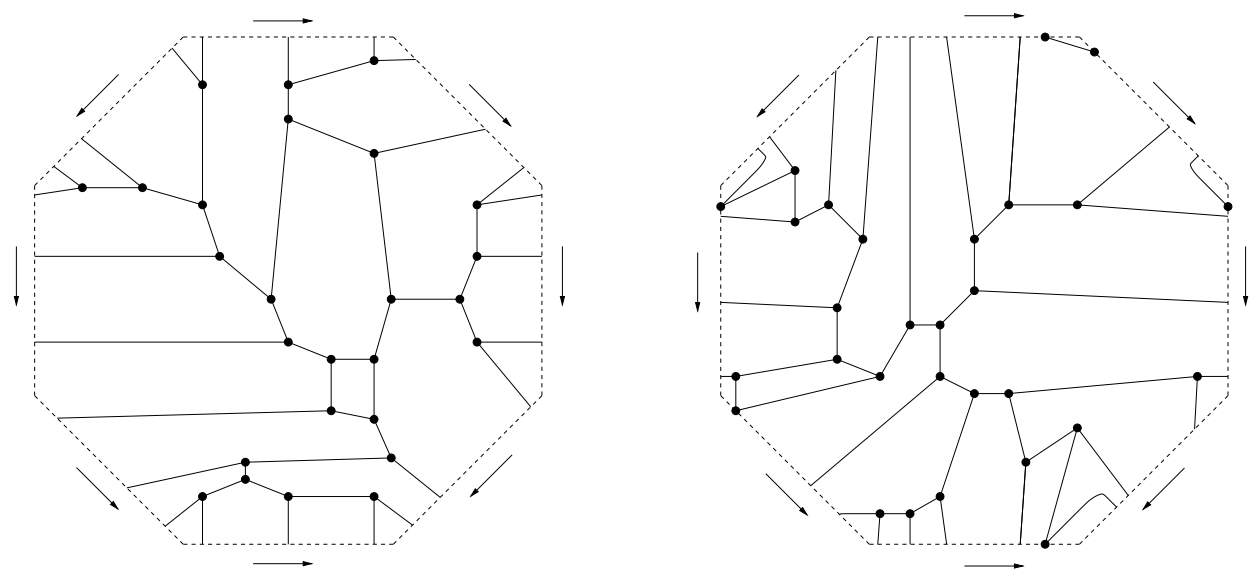

Fig. 8: The two polyhedral embeddings of genus 2 of the graph in Figure 7 Again opposite sides have to be identified.

The smallest cubic graphs that have polyhedral embeddings with different genera have 26 vertices. There are 4 of them and they all have one polyhedral embedding in the torus and two in genus 2. These graphs can be found in the graph database $H o G$ Brinkmann et al. (2013b) when searching for the keyword pol_embed. One of these graphs is shown with an embedding of genus 1 in Figure 7 and with two embeddings of genus 2 in Figure 8. There are no other polyhedral embeddings of this graph. With Corollary 14 (b) this can now be used to show that for each $k$ there is a cubic graph with embeddings in each genus $k \leq g \leq 2 k$, which implies Theorem 8 (b).

The numbers in Table 1 and Table 2 together with the fact that for different embeddings of a given graph, the number of faces decreases as the genus of the embedding increases, suggest that it is more difficult to get polyhedral embeddings for larger genera. One might even be tempted to expect that if a graph has a polyhedral embedding, it also has one of minimum genus. This is however not true and the smallest cubic counterexamples have 28 vertices. There are four of 


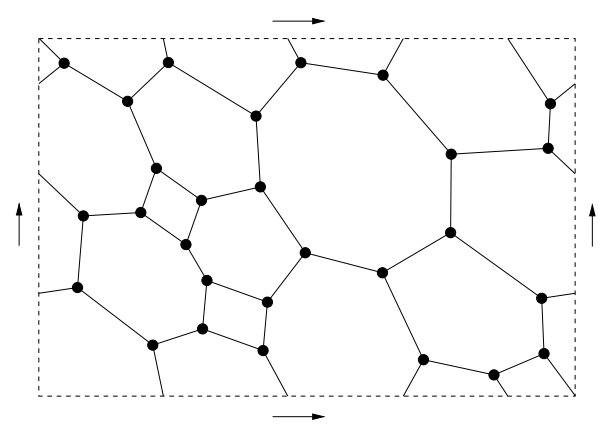

Fig. 9: A minimum genus embedding for one of the smallest cubic graphs on 28 vertices that have a polyhedral embedding, but not for their minimum genus. Again opposite sides have to be identified.

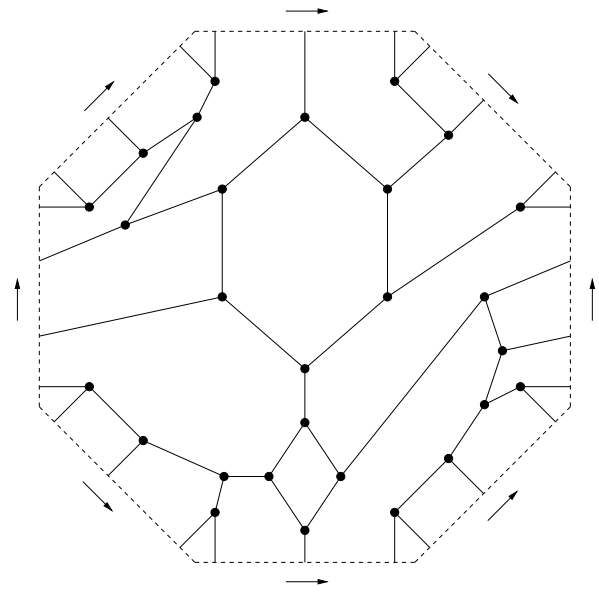

Fig. 10: A polyhedral embedding of the graph in Figure 9. Again opposite sides have to be identified.

these graphs - two have genus 1 and only one polyhedral embedding and that is in genus 2 and the other two have genus 2 and each has two polyhedral embeddings - both in genus 3. One of these counterexamples is shown in Figure 9 with a minimum genus embedding, and in Figure 10 with the unique polyhedral embedding. Also these graphs can be found in the graph database $H o G$ Brinkmann et al. (2013b) when searching for the keyword pol_embed. With Corollary 14 (a) this can now be used to show that for each $k \geq 1$ there is a cubic graph with genus $k$ whose smallest polyhedral embedding is in genus $2 k$, which implies Theorem 8 (d).

\section{Further research}

We have shown that there are cubic graphs with arbitrarily many polyhedral embeddings - in the same genus as well as in arbitrarily many different genera - but in the constructions, the genera of the graphs grow with the number of embeddings. In fact at least for embeddings in the same genus, the genus of the embeddings must grow, as - as shown by Mohar and Robertson 
Mohar and Robertson (2001) - for each genus there exists an upper bound $\zeta(2 g)$ on the number of polyhedral embeddings of a (not necessarily cubic) graph in that surface of genus $g$. The factor 2 comes from the fact that they define $\zeta()$ as a function of the Euler genus. They also show that $\zeta(2 g) \geq 48^{g}$ by using $g$ copies of $K_{7}$ attached to a plane triangulation. While in Mohar and Robertson (2001) the result is of an asymptotic nature, in Robertson et al. (2008) Robertson, Zha, and Zhao give 3 as an exact bound and characterize graphs with more than one toroidal embedding - but under the stronger condition of facewidth at least 4 . It would be very interesting to give at least a good approximation of the upper bound in the case of polyhedral embeddings of cubic graphs and small genus $g$.

(a) What is (a good approximation of) the upper bound $\zeta_{3}(2 g)$ for the number of polyhedral embeddings of a cubic graph in a surface of genus $g$ ?

For the plane we have $\zeta_{3}(0)=1$ if - as usual - we identify mirror images. The result proven in this paper implies that $\zeta_{3}(g) \geq 8^{g}$. We tested all cubic graphs on up to 28 vertices. The smallest graph with a polyhedral embedding in genus 1 has 14 vertices and - as mentioned before - already for 14 vertices there is a graph - the Heawood graph $H$ - with 8 polyhedral embeddings in the torus. Note that this is in fact the dual of the graph $K_{7}$ embedded on the torus, which was used in Mohar and Robertson (2001). For larger vertex numbers up to 28 there are many more graphs with 8 polyhedral embeddings on the torus - but no graph with more than 8 such embeddings. For genus 2 we have shown that there are cubic graphs with at least $8^{2}=64$ embeddings. The (unique) smallest cubic graph with 64 polyhedral embeddings in genus 2 has 26 vertices and is $H \star H$. For 28 vertices there are more such graphs, but none with more polyhedral embeddings. As the smallest cubic graph with a polyhedral embedding in genus 2 has 24 vertices, these numbers are too small to draw conclusions.

We know by Corollary 12 that by forming $H \star G$ for a cubic 3-connected planar graph $G$ (or even repeating this process) gives more cubic graphs with 8 polyhedral embeddings in the torus, but the reason for the comparatively large number of embeddings is rooted in $H$, so in a certain sense these graphs can be considered trivial. Due to Lemma 10 the smallest cubic graph with (at least) 8 embeddings on the torus that cannot be obtained as a star product must be cyclically 4-connected. We tested all cubic graphs with girth at least 4 on up to 30 vertices with 8 embeddings on the torus and it turned out that - except for $H$ - they all have a cyclic 3-edge-cut, so they can all be obtained from $H$ by the star product.

(b) What is the smallest cubic graph with at least 8 embeddings on the torus that cannot be obtained as a repeated star product applied to the Heawood graph?

Note that it is also possible that such a graph does not exist. E.g. in Robertson et al. (2008) the graph of the 4-by-4 grid on the torus played a special role for facewidth 4: all graphs without a unique embedding on the torus can be obtained by operations on this graph.

Though for genus 2 we do not have much data, the situation seems similar: the cubic graph with 64 embeddings in genus 2 is unique $\left(H^{2}=H \star H\right)$. On 28 vertices there are 4 graphs with 64 embeddings in genus 2: three have girth 3 (so they must be $H^{2} \star K_{4}$ ) and the other has girth 6 , but a cyclic 3 -edge-cut, so it must be $\left(H \star K_{4}\right)_{v} \star H$ with the vertex $v$ in the triangle of $H \star K_{4}$.

A question asking for a similar result to the main result in Mohar and Robertson (2001), but not fixing the genus of the embedding, but of the graph, is completely open: 
(c) Is there an upper bound $f_{a}(g)$ for the number of polyhedral embeddings of a cubic graph with genus $g$ ?

Again by Theorem $11 f_{a}(0)=1$, but the fact that already toroidal graphs can have polyhedral embeddings in the torus as well as in an arbitrarily large genus, suggests that not restricting the genus of the embedding might lead to arbitrarily many polyhedral embeddings already for the torus. Nevertheless, we know no proof of this and up to 28 vertices all graphs $G$ with the largest number of polyhedral embeddings for a fixed value of mingen $(G)$ have all embeddings in the same genus - which is in fact mingen $(G)$.

For the difference between the smallest genus that allows a polyhedral embedding and the genus of the graph, we have a similar situation: for the graphs constructed, the difference is only large for graphs that have a large genus, while even toroidal graphs can have embeddings in arbitrarily large genus. This suggests the following question:

(d) Is there an upper bound $f_{p}(g)$ on the minimum genus of a polyhedral embedding of a cubic graph $G$ with mingen $(G)=g$ that has a polyhedral embedding in some genus?

For the plane we have $f_{p}(0)=0$, but already for $g=1$ the question is open.

\section{References}

G. Brinkmann. Fast generation of cubic graphs. Journal of Graph Theory, 23(2):139-149, 1996.

G. Brinkmann. A practical algorithm for the computation of the genus. submitted, arXiv identifier 2005.08243, 2020.

G. Brinkmann, J. Goedgebeur, J. Hägglund, and K. Markström. Generation and properties of snarks. Journal of Combinatorial Theory B, 4(3):468-488, 2013a.

G. Brinkmann, J. Goedgebeur, H. Mélot, and K. Coolsaet. House of graphs: a database of interesting graphs. Discrete Applied Mathematics, 161:311-314, 2013b. http://hog.grinvin.org.

H. Van den Camp. The effect of local symmetry-preserving operations on the connectivity of embedded graphs. Master's thesis, Ghent University, 2020.

J. Gross and T. Tucker. Topological Graph Theory. John Wiley and Sons, 1987.

B. Grünbaum. Conjecture 6. In W. Tutte, editor, Recent progress in Combinatorics, page 343. Academic Press, New York, 1969. Proceedings of the Third Waterloo Conference on Combinatorics, 1968.

M. Kochol. Polyhedral embeddings of snarks in orientable surfaces. Proc. of the Am. Math. Soc., 137(5):1613-1619, 2009.

A. Kotzig. Hamilton graphs and hamilton circuits. In Theory of Graphs and its Applications, pages 63-82. Academic Press, New York, 1964. Proceedings of the Symposium held in Smolenice in June 1963. 
A. Malnič and B. Mohar. Generating locally cyclic triangulations of surfaces. J. Combin. Theory Ser. B, 56(2):147-164, 1992.

B. Mohar. Face-width of embedded graphs. Mathematica Slovaca, 47(1):35-63, 1997.

B. Mohar and N. Robertson. Flexibility of polyhedral embeddings of graphs in surfaces. $J$. Combin. Theory Ser. B, 83:38-57, 2001.

B. Mohar and C. Thomassen. Graphs on Surfaces. Johns Hopkins University Press, 2001.

B. Mohar and A. Vodopivec. On polyhedral embeddings of cubic graphs. Combinatorics Probability E Computing, 15(6):877-893, 2006.

S. Negami. Diagonal flips of triangulations on surfaces, a survey. In Proceedings of the 10th Workshop on Topological Graph Theory (Yokohama, 1998), volume 47 of Yokohama Math. J., pages 1-40, 1999.

G. Ringel and J. Youngs. Solution of the Heawood map-coloring problem. Proceedings of the National Academy of Sciences, 60(2):438-445, 1968.

N. Robertson, X. Zha, and Y. Zhao. On the flexibility of toroidal embeddings. J. Combin. Theory Ser. B, 98:43-61, 2008.

T. Sun. Face distributions of embeddings of complete graphs. J. Graph Theory, 97(2):281-304, 2021. arXiv:1708.02092v3.

C. Thomassen. Embeddings of graphs with no short noncontractible cycle. J. Combin. Theory, Ser. B, 48:155-170, 1990. 